\title{
Do fluorescent agents alter the mechanical strength of orthodontic adhesives? An in vitro and clinical study
}

\author{
Paulo Henrique Rossato ${ }^{1,2}$, Edmilson Nobumito Kaneshima², Fábio Domingues², Thais Maria Freire Fernandes ${ }^{2}$, \\ Sandrine Bittencourt Berger ${ }^{3}$ and Paula Vanessa Pedron Oltramari ${ }^{2^{*}}$ (I)
}

\begin{abstract}
Background: Fluorescent agents are added to orthodontic adhesives with the aim of making them visible under ultraviolet (UV) light, which ensures the complete, safe removal of remnants after orthodontic treatment. However, it is necessary to evaluate if the mechanical strength of these materials is maintained. Therefore, this study evaluated whether the addition of fluorescent agents influences the shear bond strength and clinical performance of a UV lightsensitive adhesive system.

Methods: This study consisted of two stages: (1) In vitro phase: 40 human teeth were selected, divided at random into 2 groups ( $n=20)$, according to the adhesive system used: UV group-adhesive with fluorescent agent, and control group - conventional adhesive. A shear bond strength test was performed using a DL 2000 universal testing machine, at a speed of $0.5 \mathrm{~mm} / \mathrm{min}$. The accessories were removed and an evaluation of the Adhesive Remnant Index (ARI) was carried out. (2) Clinical phase: 8 patients were selected and had their appliances bonded using the split-mouth design (160 teeth) with the same tested adhesive systems (UV, $n=80$; control, $n=80$ ). The patients were monitored for bonding failure for a period of 24 months. Statistical analysis was performed using the Independent $t$ test, chi-squared tests, and Mann-Whitney test, at a level of significance of $5 \%$ and confidence interval of $95 \%$.
\end{abstract}

Results: Regarding the in vitro phase, the shear bond strength test yielded similar results in the two groups $(p>0.05)$ and the ARI showed statistically significant differences between the groups with a score of 1 being the most frequent ARI for both groups (70\%). In addition, there was no clinical difference in terms of bonding failure between the groups $(p>0.05)$.

Conclusion: The addition of fluorescent elements does not alter the mechanical strength and performance of the orthodontic adhesive and represents a viable alternative for clinical application.

Keywords: Adhesive removal, Debonding, Bracket bond, UV light, Fluorescence, Shear bond strength, Adhesive remnant index

\section{Background}

The process of removing adhesive remnants after the debonding of orthodontic accessories remains a challenge for the orthodontist [1], as all the proposed techniques produce different degrees of polishing and come with some form of abrasion, accompanied by a varying degree of enamel loss [1-6]. Moreover, concerned about

\footnotetext{
* Correspondence: pvoltramari@hotmail.com

${ }^{2}$ Department of Orthodontics, UNOPAR (University of North Parana),

Londrina, Brazil

Full list of author information is available at the end of the article
}

the removal of excess adhesive, some orthodontists end up leaving remnants of material on the surface of the tooth, which promotes an accumulation of plaque and the consequent increased risk of caries [7].

Optical characteristics of natural teeth, such as fluorescence, are determined by the interaction of light with dentin, enamel, and dental pulp. This property enables the structure to absorb light energy from ultraviolet radiation and be visually differentiated, allowing contactless detection for various materials [8]. Rare earth oxides, such as europium, terbium, cerium and ytterbium, 
are well-known fluorescent materials widely used in many fields of dentistry to differentiate between dental materials and the tooth surface on visual inspection [8].

With the aim of finding more efficient solutions and making adhesives visible for complete and safe removal after orthodontic treatment, chemical elements in powder form have been added to adhesives to increase fluorescence, which permits the use of UV light for viewing the remnants after the debonding of the accessories $[9,10]$.

From a clinical perspective, recent studies [10-13] have found that the use of UV light could help orthodontists view adhesives by using fluorescent agents, since they permit a more efficient removal of the remnants after debonding (Fig. 1a, b). This technology also reduces the length of time needed to carry out the procedure of removal of the adhesive remnants [12]. Another important clinical aspect is that adhesive systems for orthodontic use require a minimum load for shear bond strength [14].

As yet, no tests have been conducted to determine if the change in the composition of the adhesive, as a result of the incorporation of fluorescent agents, could have an impact on the material's mechanical properties, such as shear bond strength, and have an adverse effect on the outcome of treatment. So, the aim of this study was to evaluate if the addition of fluorescent agents influences shear bond strength and clinical performance in a UV light-sensitive adhesive system during the debonding of orthodontic brackets.

\section{Material and methods}

The Research Ethics Committee at the UNOPAR (University of North Parana) approved the protocol of this study.

This study comprised two stages: in vitro and clinical.

For the in vitro phase, a sample size of 20 premolar teeth was considered adequate for each group, based on a previous study [10]. Thus, a total of 40 human molars, referred for extraction, were selected. The teeth were cleansed and stored at approximately $5{ }^{\circ} \mathrm{C}$, immersed in deionized distilled water, thereby preventing any interference with the adhesion mechanism, for a maximum of one week prior to the tests. The inclusion criteria were as follows: absence of visible defects or damage caused by the extraction process or cracks in the enamel, absence of white spot lesions on the enamel, absence of caries and restorations on the vestibular surface of the enamel, absence of exposure to chemical products, and full root development $[15,16]$. The root portions of the teeth were then embedded, one by one, in a PVC tube (diameter of $2 \mathrm{~cm}$ and height of $1.8 \mathrm{~cm}$ ). The inside of the tubes was filled with self-curing acrylic resin so as to maintain the tooth in a central position, with the crown


Fig. 1 Clinical perspective of adhesive remnant after debonding with UV (a) and conventional (b) adhesives

free, in order to permit the bonding of the accessories and position them with the vestibular surface in the perpendicular position. After the polymerization of the acrylic resin, the vestibular surfaces of all the samples were subjected to prophylaxis with a paste of pumicestone and water, with the aid of a rubber cup, at low rotation for $10 \mathrm{~s}$. The rubber cups were replaced every 5 teeth, which provided standardization in terms of the mechanical action of the prophylaxis for all the teeth. The teeth were then washed in running water for $10 \mathrm{~s}$, and subsequently dried with light jets of oil-free, compressed air for $20 \mathrm{~s}$ [17]. The teeth received orthodontic tubes (3M Oral Care, Monrovia, CA, USA), bonded using the following adhesive systems: UV group ( $n=$ 20), Orthocem UV Trace adhesive with fluorescent agent (FGM, Joinville, Santa Catarina, Brazil); C group $(n=20)$, conventional adhesive (Transbond XT, 3M Oral Care). The accessories were cemented according to manufacturers' instructions, as described in Table 1.

All the adhesive systems were polymerized using an SDI Radii-Cal curing light, with a peak light intensity of $1200 \mathrm{~mW} / \mathrm{cm}^{2}$ (Dental Limited, Bayswater, Victoria, Australia), observing the manufacturer's instructions. A single operator bonded all orthodontic tubes with a quantity of composite resin sufficient to cover the entire base of the accessories. The bonding was carried out in order to enable a careful fitting of the tubes in the center of the clinical crowns of the teeth (final position), applying firm pressure, so that the accessories were seated perfectly at the enamel surface. The excess adhesive around the entire base of these tubes was then removed 
Table 1 Composition of the resins investigated

\begin{tabular}{|c|c|c|c|}
\hline Material & Manufacturer & Composition: (\% weight) & Application \\
\hline $\begin{array}{l}\text { Orthocem UV (UV } \\
\text { group) }\end{array}$ & $\begin{array}{l}\text { Dentscare Ltda. } \\
\text { (Joinville, Brazil) }\end{array}$ & $\begin{array}{l}\text {-Bisphenol A Diglycidyl ether } \\
\text { methacrylate (BisGMA): } 25-35 \\
\text {-Triethylene glycoldimethacrylate } \\
\text { (TEGDMA): } 10-15 \\
\text {-Phosphate methacrylate } \\
\text { monomer: }>2 \\
\text {-Silanized silicon dioxide: } 45-60 \\
\text {-Camphorquinone: < } 1 \\
\text {-Sodium fluoride: > 1 } \\
\text {-Fluorescent pigment: }<0.01\end{array}$ & $\begin{array}{l}\text {-Etching with } 37 \% \text { phosphoric acid for } 15 \mathrm{~s} ; \\
\text {-Continuous jet of water for } 20 \mathrm{~s} \text {; } \\
\text { - Oil- and moisture-free jets of air; } \\
\text {-Composite resin applied to the base of orthodontic accessory; } \\
\text {-Polymerization for } 20 \mathrm{~s} \text { (10 s mesial and } 10 \mathrm{~s} \text { distal). }\end{array}$ \\
\hline $\begin{array}{l}\text { Transbond XT (C } \\
\text { group) }\end{array}$ & $\begin{array}{l}\text { 3M Oral Care } \\
\text { (Monrovia, CA, USA) }\end{array}$ & $\begin{array}{l}\text {-Organic matrix: Bis-GMA and } \\
\text { TEGDMA } \\
\text {-Inorganic part: silica silanized } \\
\text { with } 70 \text { to } 80 \% \text { by volume } \\
\text {-n-dimethyl benzocaine, } \\
\text { hexafluorphosphate } \\
\text {-Camphorquinone }\end{array}$ & $\begin{array}{l}\text {-Etching with } 37 \% \text { phosphoric acid for } 15 \mathrm{~s} ; \\
\text {-Continuous jet of water for } 20 \mathrm{~s} \text {; } \\
\text {-Oil- and moisture-free jets of air; } \\
\text {-Application of Transbond XT Primer adhesive (3M, Monrovia, CA, USA) } \\
\text { and photo-polymerized for } 30 \mathrm{~s} \text {; } \\
\text {-Application of Transbond XT at the base of the orthodontic accessory, } \\
\text { which was polymerized for } 20 \mathrm{~s} \text { (10 s mesial and } 10 \mathrm{~s} \text { distal). }\end{array}$ \\
\hline
\end{tabular}

$[15,18]$. After bonding, the samples were stored in distilled water and kept in a $37{ }^{\circ} \mathrm{C}$ oven for $24 \mathrm{~h}$ [19]. At the end of this period, the test specimens were fitted in a cylinder with jaw clamps regulating their position so that the pivot point of the machine would execute a movement parallel to the vestibular surface of the teeth. The shearing tool was placed at the tube/enamel interface. The shear bond strength test was performed using a universal testing machine (DL 2000 EMIC), set to a speed of $0.5 \mathrm{~mm} / \mathrm{min}$, until the accessories were removed. The test results were obtained in $\mathrm{N}$ (Newtons) and converted to pressure values in MPa (Megapascal), verified based on the measurement of the base of the bracket used. After shearing, the vestibular surfaces of the samples were evaluated by two examiners (neither of whom were involved in the bonding procedure), in a blind analysis, in order to evaluate the Adhesive Remnant Index (ARI) [20] using the following system of scoring: 0-no adhesive remaining, 1-less than half of adhesive remaining, 2-more than half of adhesive remaining, 3-all adhesive remaining on the tooth surface. The viewing and evaluation of the tooth surfaces were carried out with the aid of a BVM100 microscope (BEL Photonics, Italy), at a magnification of up to $\times 10$. Examiner calibration was carried out by means of repeated sample evaluation. The result was obtained by means of the consensus of the two examiners.

For the clinical phase, 8 patients (mean age $23.1 \pm 2.3$; 6 males and 2 females) requiring fixed orthodontic appliances were randomly enrolled. The inclusion criteria included class I malocclusion, absence of visible defects, damage or fissures, white spot lesions, caries, and restorations on the surface of the buccal enamel. Exclusion criteria were as follows: missing permanent teeth, supernumerary teeth, and prior orthodontic treatment. The study was based on a double-blinded split-mouth design, and the two adhesives were randomly applied on each side. All patients were blinded to the allocation of treatment and received brackets (3M Oral Care) that were bonded on one side with Orthocem UV Trace (FGM) (UV group, $n=80$ ) adhesive with fluorescent agent, and on the other side with Transbond XT (3M/Oral Care) (control group), $n=80$ ). The same operator bonded all the brackets, applying the same bonding technique, following manufacturers' instructions (Table 1). It was not possible to blind the operators to the bonding system used because the two systems employed different methods of application. Patients were monitored over a 24-month period using the same wire sequence. A blinded external examiner assessed bonding failures for the data analysis.

\section{Statistical analysis}

The laboratory results were analyzed through the independent $t$ test. Clinical comparisons of bonding failures were analyzed via the chi-squared test with Yates correction with the Mann-Whitney test being used for assessing the ARI. All tests were analyzed at a level of significance of $5 \%$ and confidence interval of $95 \%$, using BioEstat software.

\section{Results}

With regard to the shear bond strength test, both adhesives produced similar results $(p>0.05)$ (Table 2).

Figure 2 shows the observed frequency of ARI scores for each adhesive system tested. The test showed statistically significant differences in the ARI scores between the groups $(p=0.022)$. In the groups tested, no score 3 was observed and the highest frequency noted was for score 1 (70\%).

Clinical results presented very low occurrences of failure (Table 3), with no significant difference between the groups. Bond failure occurred in $2.5 \%$ for the UV group 
Table 2 Shear bond strength (MPa) for both groups: mean, standard deviation (SD) and independent $t$ test $(P)$

\begin{tabular}{|c|c|c|c|c|}
\hline \multicolumn{5}{|c|}{ Shear bond strength (MPa) } \\
\hline \multicolumn{2}{|c|}{$\begin{array}{l}\text { UVgroup } \\
(\mathrm{n}=30)\end{array}$} & \multicolumn{2}{|c|}{$\begin{array}{l}\text { Control group } \\
(n=30)\end{array}$} & \multirow[t]{2}{*}{$p$} \\
\hline Mean & SD & Mean & SD & \\
\hline 12.69 & 5.05 & 13.80 & 4.55 & $>0.05$ \\
\hline
\end{tabular}

and $5 \%$ for the control group, although this difference was not statistically significant $(p>0.05)$.

\section{Discussion}

It is known that all techniques employed for removing adhesive remnants (AR), following the debonding of brackets, produce different degrees of polishing and introduce some form of abrasion, accompanied by various degrees of enamel loss [1-6]. Thus, the quest for a safe, efficient method for the removal of AR, after the debonding of orthodontic accessories, has resulted in the introduction of a wide variety of instruments and procedures [1, 4, 21-23]. One possibility is the incorporation of fluorescent agents into adhesive systems capable of allowing the clinician to check the differences in fluorescence between the adhesive material and the enamel, thereby preserving the tooth surface [9, 13, 24, 25].

However, an important step in the verification of the efficiency of an adhesive system, for use in orthodontics, is the shear bond strength test [26, 27]. In the present study, this aspect was evaluated with particular attention due to the presence of an adhesive system with fluorescent agents (Orthocem UV trace, UV group), since the other one is a widely known system already used by professionals $[28,29]$. The results demonstrated that the adhesive systems used did not show significant differences in terms of shear bond strength, with values close to the results previously described in the literature [28]. It should be emphasized that, despite the Orthocem UV Trace (UV group) being a two-step system, since it does not include the adhesive stage, this did not have an adverse effect on performance. Moreover, the presence of fluorescent agents in the UV group adhesive, similarly, had no impact on performance.

Rare earth oxides, widely used in dentistry to differentiate between dental materials and the tooth surface on visual inspection [8], have also been used to benefit orthodontic patients. The $\mathrm{Eu}^{3+}$ ion, one of the most popular lanthanides, is useful for the development of fluorescent, orthodontic adhesives as it emits monochromatic red light when excited with ultraviolet (UV) light. With the aim of looking for more effective solutions and making adhesives visible, for safe, complete removal after orthodontic treatment, Hamba et al. [9] added $\mathrm{Eu}^{3+}$ ions to yttrium oxide $\left(\mathrm{Y}^{2} \mathrm{O}^{3}\right)$, which was a viable alternative for differentiation. Namura et al. [30], looked into the effect of incorporating different concentrations $(0.001 \%, 0.002 \%$, and $0.003 \%)$ of fluorescent dye derived from coumarin, in an adhesive system for bonding brackets to bovine teeth, and concluded that the adhesive containing $0.002 \%$ fluorescent dye possessed good shear bond strength $(6.6 \mathrm{MPa})$, and also facilitated the removal of remaining material without impairing the structure of the enamel. The present study employed the UV system, though the manufacturer does not state what fluorescent agent is used, just the concentration (< $0.001 \%$ by mass). Therefore, this study obtained a shear bond strength greater than that of the abovementioned study [30] for all the adhesives tested, probably due to the bonding having been performed on human teeth.




Table 3 Clinical performance (success and failure) in clinical bonding for UV group and control group: $n$ and frequency (\%) of occurrence, chi-squared test ( $p$ )

\begin{tabular}{llll}
\hline Group & $\begin{array}{l}\text { Success } \\
n(\%)\end{array}$ & $\begin{array}{l}\text { Failure } \\
n(\%)\end{array}$ & $\begin{array}{l}\text { Total } \\
n(\%)\end{array}$ \\
\hline UV & $78(97.5)$ & $2(2.5)$ & $80(100)$ \\
$C$ & $76(95)$ & $4(5)$ & $80(100)$ \\
$p$ & $>0.05$ & & \\
\hline
\end{tabular}

The present study showed significant differences between groups for the ARI scores. Nevertheless, both groups exhibited higher frequencies of score 1 for the ARI (70\%) in all systems tested, representing less than half of the adhesive remaining on the tooth surface after debonding, constituting a clinically desirable result because it reduced the amount of residual adhesive and the need for a rotary instrument for the cleanup [31]. However, a previous clinical study demonstrated that enamel damage regularly occurred during the debonding process with the extent of the damage being highly variable depending on the bracket material and adhesive system [32].

The laboratory results for both tested adhesives produced values above the recommended average, between 6 and $8 \mathrm{MPa}$, which represents the minimum load for the shear bond strength of an adhesive for clinical use in orthodontics [14]. It should also be stressed that highly accentuated shear bond strength could increase the risk of the enamel fracturing during the removal of the orthodontic accessories. Therefore, a suitable adhesive system for orthodontic use must be seen to be resistant to masticatory force [14] while preserving the dental structure in the accessory removal stage [32].

Additionally, the clinical results showed low failure rates for both adhesive systems tested (UV group = $2.5 \%$ and control group $=5 \%$ ). Cal-Neto et al. [33] suggested that a failure rate below $10 \%$ is clinically acceptable. The UV adhesive system was compared to the gold standard in orthodontics and demonstrated similar clinical performance over a 24-month period. Despite the lack of evaluation at the end of the treatment, which is a limitation of the current study, the study was conducted considering that an average treatment time is 23.5 months [34].

Accordingly, this study compared a UV light-sensitive adhesive system to a conventional system, demonstrating no significant difference in shear bond strength and in clinical performance. These results show that the adhesive system with the fluorescent agent can be used and could deliver greater clinical practicality in the removal of adhesive remnants, particularly in posterior teeth, due to the differentiation of the optical characteristics of natural teeth. These characteristics could prevent potential damage to the surface layer of the enamel. Further investigation could be performed comparing the current adhesive with the fluorescent substance and the same material without it. This evaluation could address important information regarding the influence of fluorescent agents on the mechanical strength of orthodontic adhesives.

\section{Conclusion}

- The addition of fluorescent elements does not alter the in vitro and clinical mechanical strength of the orthodontic adhesive;

- Adhesive systems with fluorescent agents represent a viable alternative for orthodontic use.

\section{Abbreviations}

AR: Adhesive remnant; ARI: Adhesive Remnant Index; CA: California; USA: United States of America; UV: Ultraviolet

\section{Acknowledgements}

Not applicable

Authors' contributions

PHR carried out the data analysis and manuscript editing. ENK participated in the laboratorial data acquisition and manuscript correction. FD conducted all clinical phases. TMFF participated in the manuscript correction. SBB participated in the design of the study and manuscript correction. PVPO participated in the design of the study, organized the laboratorial and clinical phases and manuscript correction. All authors read and approved the final manuscript.

\section{Funding}

Supported by the Fundação Nacional de Desenvolvimento do Ensino Superior Particular (FUNADESP)

\section{Availability of data and materials}

All data generated or analyzed during this study are included in this published article. Please contact the corresponding author for requests for data.

Ethical approval and consent to participate

Prior to commencing this study, the protocol was approved by the Research Ethics Committee at UNOPAR (University of North Parana) (Opinion no. 846.387; CAAE: 33345114.2.0000.0108).

\section{Consent for publication}

Consent was obtained from the patient prior to the study commencing, or in the case of children, from their parent or legal guardian.

\section{Competing interests}

The authors declare that they have no competing interests.

\section{Author details}

${ }^{1}$ Department of Oral Health, IFPR, Federal Institute of Parana (IFPR), Londrina, Brazil. 2Department of Orthodontics, UNOPAR (University of North Parana), Londrina, Brazil. ${ }^{3}$ Department of Restorative Dentistry, UNOPAR (University of North Parana), Londrina, Brazil.

Received: 26 September 2019 Accepted: 6 January 2020

Published online: 10 February 2020

\section{References}

1. Hosein I, Sheriff M, Ireland AJ. Enamel loss during bonding, debonding, and cleanup with use of a self-etching primer. Am J Orthod Dentof Orthop. 2004;126(6):717-24. 
2. Al Shamsi AH, Cunningham JL, Lamey PJ, Lynch E. Three-dimensional measurement of residual adhesive and enamel loss on teeth after debonding of orthodontic brackets: an in-vitro study. Am J Orthod Dentof Orthop. 2007;131(3):301 e9-15.

3. Ryf S, Flury S, Palaniappan S, Lussi A, van Meerbeek B, Zimmerli B. Enamel loss and adhesive remnants following bracket removal and various clean-up procedures in vitro. Eur J Orthod. 2012;34(1):25-32.

4. Krell KV, Courey J, Bishara SE. Orthodontic bracket removal using conventional and ultrasonic debonding techniques, enamel loss, and time requirements. Am J Orthod Dentof Orthop. 1993;103(3):258-66.

5. Janiszewska-Olszowska J, Tandecka K, Szatkiewicz T, Sporniak-Tutak K, Grocholewicz K. Three-dimensional quantitative analysis of adhesive remnants and enamel loss resulting from debonding orthodontic molar tubes. Head Face Med. 2014;10:37.

6. Ulusoy C. Comparison of finishing and polishing systems for residual resin removal after debonding. J Appl Oral Sci. 2009;17(3):209-15.

7. Ahrari F, Akbari M, Akbari J, Dabiri G. Enamel surface roughness afte debonding of orthodontic brackets and various clean-up techniques. J Dent. 2013;10(1):82-93.

8. Uo M, Okamoto M, Watari F, Tani K, Morita M, Shintani A. Rare earth oxidecontaining fluorescent glass filler for composite resin. Dent Mater J. 2005; 24(1):49-52.

9. Hamba Y, Yamagata S, Akasaka T, Uo M, lida J, Watari F. Preparation and properties of fluorescent orthodontic adhesives containing Y2O3:Eu3+ particles. Nano Biomed Eng. 2013;5(2):75-84.

10. Ribeiro AA, Almeida LF, Martins LP, Martins RP. Assessing adhesive remnant removal and enamel damage with ultraviolet light: An in-vitro study. Am J Orthod Dentof Orthop. 2017;151(2):292-6.

11. Rocha RS, Salomao FM, Machado LS, Sundfeld RH, Fagundes TC. Efficacy of auxiliary devices for removal of fluorescent residue after bracket debonding. Angle Orthod. 2017:87(3):440-7

12. Kaneshima EN, Berger SB, Fernandes TMF, Navarro MFL, Oltramari-Navarro PVP. Using UV light for adhesive remnant removal after debonding of orthodontic accessories. Braz Oral Res. 2018;32:0047.

13. Lai C, Bush PJ, Warunek S, Covell DA, Al-Jewair T. An in vitro comparison of ultraviolet versus white light in the detection of adhesive remnants during orthodontic debonding. Angle Orthod. 2019;89(3):438-45.

14. Littlewood SJ, Mitchell L, Greenwood DC. A randomized controlled trial to investigate brackets bonded with a hydrophilic primer. J Orthod. 2001;28(4):301-5.

15. Ferreira FG, Nouer DF, Silva NP, Garbui IU, Correr-Sobrinho L, Nouer PRA Qualitative and quantitative evaluation of human dental enamel after bracket debonding: a noncontact three-dimensional optical profilometry analysis. Clin Oral Invest. 2014;18:1853-64.

16. Karan S, Kircelli BH, Tasdelen B. Enamel surface roughness after debonding. Angle Orthod. 2010;80(6):1081-8.

17. De Marchi R, De Marchi L, Terada RSS, Terada HH. Comparison between two methods for resin removing after bracket debonding. Dental Press $J$ Orthod. 2012;17(6):130-6.

18. Ireland AJ, Hosein I, Sherriff M. Enamel loss at bond-up, debond and cleanup following the use of a conventional light-cured composite and a resinmodified glass polyalkenoate cement. Eur J Orthod. 2005:27(4):413-9.

19. Johnston CD, Burden DJ, Hussey DL, Mitchell CA. Bonding to molars-the effect of etch time (an in vitro study). Eur J Orthod. 1998:20:195-9.

20. Artun J, Bergland S. Clinical trials with crystal growth conditioning as an alternative to acid-etch enamel pretreatment. Am J Orthod. 1984;85(4):333-40.

21. Campbell P. Enamel surfaces after orthodontic bracket debonding. Angle Orthod. 1995;65(2):103-10.

22. Retief $D H$, Denys FR. Finishing of enamel surfaces after debonding of orthodontic attachments. Angle Orthod. 1979;49(1):1-10.

23. Albuquerque GS, Vedovello Filho M, Lucato AS, Boeck EM, Degan V, Kuramae M. Evaluation of enamel roughness after ceramic bracket debonding and clean-up with different methods. Braz J Oral Sci. 2010;9:81-4.

24. Yamagata S, Iwasaki H, Hamba Y, Nakanishi K, Ushijima N, Abe S, et al. An evaluation of fluorescent orthodontic adhesives contining Eu-doped $\mathrm{ZnO}$ at room temperature. Nano Biomed Eng. 2013;5(1):31-8.

25. Yamagata S, Hamba Y, Nakanishi K, Abe S, Akasaka T, Ushijima N, Uo M, Ilda J, Watari F. Introducion of Rare-Earth-Element-Containing ZnO Nanoparticles into Orthodontic Adhesives. Nano Biomed Eng. 2012;4(1):11-7.

26. Sharma S, Tandon P, Nagar A, Singh GP, Singh A, Chugh VK. A comparison of shear bond strength of orthodontic brackets bonded with four different orthodontic adhesives. J Orthod Sci. 2014;3(2):29-33.
27. Bakhadher W, Halawany H, Talic N, Abraham N, Jacob V. Factors affecting the shear bond strength of orthodontic brackets - a review of in vitro studies. Acta Medica. 2015;58(2):43-8.

28. Turkkahraman $\mathrm{H}$, Adanir $\mathrm{N}$, Gungor AY, Alkis $\mathrm{H}$. In vitro evaluation of shear bond strengths of colour change adhesives. Eur J Orthod. 2010;32(5):571-4.

29. Verma G, Trehan M, Sharma S. Comparison of shear bond strength and estimation of adhesive remnant index between light-cure composite and dual-cure composite: an in vitro study. Int J Clin Pediatr Dent. 2013;6(3):166-70.

30. Namura Y, Tsuruoka T, Ryu C, Kaketani M, Shimizu N. Usefulness of orthodontic adhesive-containing fluorescent dye. Eur J Orthod. 2010;32(6):620-6.

31. Faria Junior EM, Guiraldo R, Berger SB, Correr AB, Correr-Sobrinho L, Contreras EF, Lopes MB. In-vivo evaluation of the surface roughness and morphology of enamel after bracket removal and polishing by different techniques. Am J Orthod Dentof Orthop. 2015;147:324-9.

32. Cochrane NJ, Lo TWG, Adams GG, Schneider PM. Quantitative analysis of enamel on debonded orthodontic brackets. Am J Orthod Dentof Orthop. 2017;152(3):312-9.

33. Cal-Neto JP, Miguel JA. An in vivo evaluation of bond failure rates with hydrophilic and self-etching primer systems. J Clin Orthod. 2005;39(12):701-2

34. Skidmore KJ, Brook KJ, Thomson WM, Harding WJ. Factors influencing treatment time in orthodontic patients. Am J Orthod Dentof Orthop. 2006; 129(2):230-8.

\section{Publisher's Note}

Springer Nature remains neutral with regard to jurisdictional claims in published maps and institutional affiliations.

\section{Submit your manuscript to a SpringerOpen ${ }^{\circ}$ journal and benefit from:}

- Convenient online submission

- Rigorous peer review

- Open access: articles freely available online

High visibility within the field

- Retaining the copyright to your article

Submit your next manuscript at $>$ springeropen.com 